\title{
Polymer Quantum Mechanics and its Continuum Limit
}

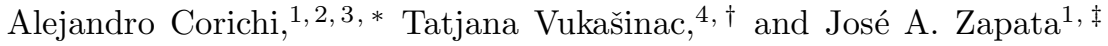 \\ ${ }^{1}$ Instituto de Matemáticas, Unidad Morelia, Universidad Nacional Autónoma de México, \\ UNAM-Campus Morelia, A. Postal 61-3, Morelia, Michoacán 58090, Mexico \\ ${ }^{2}$ Instituto de Ciencias Nucleares, Universidad Nacional Autónoma de México, \\ A. Postal 70-543, México D.F. 04510, Mexico \\ ${ }^{3}$ Institute for Gravitational Physics and Geometry, Physics Department, \\ Pennsylvania State University, University Park PA 16802, USA \\ ${ }^{4}$ Facultad de Ingeniería Civil, Universidad Michoacana de San Nicolas de Hidalgo, \\ Morelia, Michoacán 58000, Mexico
}

\begin{abstract}
A rather non-standard quantum representation of the canonical commutation relations of quantum mechanics systems, known as the polymer representation has gained some attention in recent years, due to its possible relation with Planck scale physics. In particular, this approach has been followed in a symmetric sector of loop quantum gravity known as loop quantum cosmology. Here we explore different aspects of the relation between the ordinary Schrödinger theory and the polymer description. The paper has two parts. In the first one, we derive the polymer quantum mechanics starting from the ordinary Schrödinger theory and show that the polymer description arises as an appropriate limit. In the second part we consider the continuum limit of this theory, namely, the reverse process in which one starts from the discrete theory and tries to recover back the ordinary Schrödinger quantum mechanics. We consider several examples of interest, including the harmonic oscillator, the free particle and a simple cosmological model.

PACS numbers: 04.60.Pp, 04.60.Ds, 04.60.Nc 11.10.Gh.
\end{abstract}

\section{INTRODUCTION}

The so-called polymer quantum mechanics, a nonregular and somewhat 'exotic' representation of the canonical commutation relations (CCR) [1], has been used to explore both mathematical and physical issues in background independent theories such as quantum gravity [2, 3]. A notable example of this type of quantization, when applied to minisuperspace models has given way to what is known as loop quantum cosmology [4, 5]. As in any toy model situation, one hopes to learn about the subtle technical and conceptual issues that are present in full quantum gravity by means of simple, finite dimensional examples. This formalism is not an exception in this regard. Apart from this motivation coming from physics at the Planck scale, one can independently ask for the relation between the standard continuous representations and their polymer cousins at the level of mathematical physics. A deeper understanding of this relation becomes important on its own.

The polymer quantization is made of several steps. The first one is to build a representation of the Heisenberg-Weyl algebra on a Kinematical Hilbert space that is "background independent", and that is sometimes referred to as the polymeric Hilbert space $\mathcal{H}_{\text {poly }}$. The second and most important part, the implementation of dynamics, deals with the definition of a Hamiltonian (or Hamiltonian constraint) on this space. In the examples

\footnotetext{
*Electronic address: corichi@matmor.unam.mx

${ }^{\dagger}$ Electronic address: tatjana@shi.matmor.unam.mx

${ }^{\ddagger}$ Electronic address: zapata@matmor.unam.mx
}

studied so far, the first part is fairly well understood, yielding the kinematical Hilbert space $\mathcal{H}_{\text {poly }}$ that is, however, non-separable. For the second step, a natural implementation of the dynamics has proved to be a bit more difficult, given that a direct definition of the Hamiltonian $\hat{H}$ of, say, a particle on a potential on the space $\mathcal{H}_{\text {poly }}$ is not possible since one of the main features of this representation is that the operators $\hat{q}$ and $\hat{p}$ cannot be both simultaneously defined (nor their analogues in theories involving more elaborate variables). Thus, any operator that involves (powers of) the not defined variable has to be regulated by a well defined operator which normally involves introducing some extra structure on the configuration (or momentum) space, namely a lattice. However, this new structure that plays the role of a regulator can not be removed when working in $\mathcal{H}_{\text {poly }}$ and one is left with the ambiguity that is present in any regularization. The freedom in choosing it can be sometimes associated with a length scale (the lattice spacing). For ordinary quantum systems such as a simple harmonic oscillator, that has been studied in detail from the polymer viewpoint, it has been argued that if this length scale is taken to be 'sufficiently small', one can arbitrarily approximate standard Schrödinger quantum mechanics [2, 3]. In the case of loop quantum cosmology, the minimum area gap $A_{0}$ of the full quantum gravity theory imposes such a scale, that is then taken to be fundamental [4].

A natural question is to ask what happens when we change this scale and go to even smaller 'distances', that is, when we refine the lattice on which the dynamics of the theory is defined. Can we define consistency conditions between these scales? Or even better, can we take the limit and find thus a continuum limit? As it 
has been shown recently in detail, the answer to both questions is in the affirmative [6]. There, an appropriate notion of scale was defined in such a way that one could define refinements of the theory and pose in a precise fashion the question of the continuum limit of the theory. These results could also be seen as handing a procedure to remove the regulator when working on the appropriate space. The purpose of this paper is to further explore different aspects of the relation between the continuum and the polymer representation. In particular in the first part we put forward a novel way of deriving the polymer representation from the ordinary Schrödinger representation as an appropriate limit. In Sec. II we derive two versions of the polymer representation as different limits of the Schrödinger theory. In Sec. III we show that these two versions can be seen as different polarizations of the 'abstract' polymer representation. These results, to the best of our knowledge, are new and have not been reported elsewhere. In Sec. IV we pose the problem of implementing the dynamics on the polymer representation. In Sec. $[\nabla]$ we motivate further the question of the continuum limit (i.e. the proper removal of the regulator) and recall the basic constructions of [6]. Several examples are considered in Sec. VI In particular a simple harmonic oscillator, the polymer free particle and a simple quantum cosmology model are considered. The free particle and the cosmological model represent a generalization of the results obtained in [6] where only systems with a discrete and non-degenerate spectrum where considered. We end the paper with a discussion in Sec. VII. In order to make the paper self-contained, we will keep the level of rigor in the presentation to that found in the standard theoretical physics literature.

\section{QUANTIZATION AND POLYMER REPRESENTATION}

In this section we derive the so called polymer representation of quantum mechanics starting from a specific reformulation of the ordinary Schrödinger representation. Our starting point will be the simplest of all possible phase spaces, namely $\Gamma=\mathbb{R}^{2}$ corresponding to a particle living on the real line $\mathbb{R}$. Let us choose coordinates $(q, p)$ thereon. As a first step we shall consider the quantization of this system that leads to the standard quantum theory in the Schrödinger description. A convenient route is to introduce the necessary structure to define the Fock representation of such system. From this perspective, the passage to the polymeric case becomes clearest. Roughly speaking by a quantization one means a passage from the classical algebraic bracket, the Poisson bracket,

$$
\{q, p\}=1
$$

to a quantum bracket given by the commutator of the corresponding operators,

$$
[\hat{q}, \hat{p}]=i \hbar \hat{\mathbf{1}}
$$

These relations, known as the canonical commutation relation (CCR) become the most common corner stone of the (kinematics of the) quantum theory; they should be satisfied by the quantum system, when represented on a Hilbert space $\mathcal{H}$.

There are alternative points of departure for quantum kinematics. Here we consider the algebra generated by the exponentiated versions of $\hat{q}$ and $\hat{p}$ that are denoted by,

$$
U(\alpha)=e^{i(\alpha \hat{q}) / \hbar} \quad ; \quad V(\beta)=e^{i(\beta \hat{p}) / \hbar}
$$

where $\alpha$ and $\beta$ have dimensions of momentum and length, respectively. The CCR now become

$$
U(\alpha) \cdot V(\beta)=e^{(-i \alpha \beta) / \hbar} V(\beta) \cdot U(\alpha)
$$

and the rest of the product is

$$
U\left(\alpha_{1}\right) \cdot U\left(\alpha_{2}\right)=U\left(\alpha_{1}+\alpha_{2}\right) \quad ; \quad V\left(\beta_{1}\right) \cdot V\left(\beta_{2}\right)=V\left(\beta_{1}+\beta_{2}\right)
$$

The Weyl algebra $\mathcal{W}$ is generated by taking finite linear combinations of the generators $U\left(\alpha_{i}\right)$ and $V\left(\beta_{i}\right)$ where the product (3) is extended by linearity,

$$
\sum_{i}\left(A_{i} U\left(\alpha_{i}\right)+B_{i} V\left(\beta_{i}\right)\right)
$$

From this perspective, quantization means finding an unitary representation of the Weyl algebra $\mathcal{W}$ on a Hilbert space $\mathcal{H}^{\prime}$ (that could be different from the ordinary Schrödinger representation). At first it might look weird to attempt this approach given that we know how to quantize such a simple system; what do we need such a complicated object as $\mathcal{W}$ for? It is infinite dimensional, whereas the set $\mathcal{S}=\{\hat{\mathbf{1}}, \hat{q}, \hat{p}\}$, the starting point of the ordinary Dirac quantization, is rather simple. It is in the quantization of field systems that the advantages of the Weyl approach can be fully appreciated, but it is also useful for introducing the polymer quantization and comparing it to the standard quantization. This is the strategy that we follow.

A question that one can ask is whether there is any freedom in quantizing the system to obtain the ordinary Schrödinger representation. On a first sight it might seem that there is none given the Stone-Von Neumann uniqueness theorem. Let us review what would be the argument for the standard construction. Let us ask that the representation we want to build up is of the Schrödinger type, namely, where states are wave functions of configuration space $\psi(q)$. There are two ingredients to the construction of the representation, namely the specification of how the basic operators $(\hat{q}, \hat{p})$ will act, and the nature of the space of functions that $\psi$ belongs to, that is normally fixed by the choice of inner product on $\mathcal{H}$, or measure $\mu$ on $\mathbb{R}$. The standard choice is to select the Hilbert space to be,

$$
\mathcal{H}=L^{2}(\mathbb{R}, \mathrm{d} q)
$$


the space of square-integrable functions with respect to the Lebesgue measure $\mathrm{d} q$ (invariant under constant translations) on $\mathbb{R}$. The operators are then represented as,

$$
\hat{q} \cdot \psi(q)=(q \psi)(q) \quad \text { and } \quad \hat{p} \cdot \psi(q)=-i \hbar \frac{\partial}{\partial q} \psi(q)
$$

Is it possible to find other representations? In order to appreciate this freedom we go to the Weyl algebra and build the quantum theory thereon. The representation of the Weyl algebra that can be called of the 'Fock type' involves the definition of an extra structure on the phase space $\Gamma$ : a complex structure $J$. That is, a linear mapping from $\Gamma$ to itself such that $J^{2}=-1$. In 2 dimensions, all the freedom in the choice of $J$ is contained in the choice of a parameter $d$ with dimensions of length. It is also convenient to define: $k=p / \hbar$ that has dimensions of $1 / L$. We have then,

$$
J_{d}:(q, k) \mapsto\left(-d^{2} k, q / d^{2}\right)
$$

This object together with the symplectic structure: $\Omega\left((q, p) ;\left(q^{\prime}, p^{\prime}\right)\right)=q p^{\prime}-p q^{\prime}$ define an inner product on $\Gamma$ by the formula $g_{d}(\cdot ; \cdot)=\Omega\left(\cdot ; J_{d} \cdot\right)$ such that:

$$
g_{d}\left((q, p) ;\left(q^{\prime}, p^{\prime}\right)\right)=\frac{1}{d^{2}} q q^{\prime}+\frac{d^{2}}{\hbar^{2}} p p^{\prime}
$$

which is dimension-less and positive definite. Note that with this quantities one can define complex coordinates $(\zeta, \bar{\zeta})$ as usual:

$$
\zeta=\frac{1}{d} q+i \frac{d}{\hbar} p \quad ; \quad \bar{\zeta}=\frac{1}{d} q-i \frac{d}{\hbar} p
$$

from which one can build the standard Fock representation. Thus, one can alternatively view the introduction of the length parameter $d$ as the quantity needed to define (dimensionless) complex coordinates on the phase space. But what is the relevance of this object ( $J$ or d)? The definition of complex coordinates is useful for the construction of the Fock space since from them one can define, in a natural way, creation and annihilation operators. But for the Schrödinger representation we are interested here, it is a bit more subtle. The subtlety is that within this approach one uses the algebraic properties of $\mathcal{W}$ to construct the Hilbert space via what is known as the Gel'fand-Naimark-Segal (GNS) construction. This implies that the measure in the Schrödinger representation becomes non trivial and thus the momentum operator acquires an extra term in order to render the operator self-adjoint. The representation of the Weyl algebra is then, when acting on functions $\phi(q)$ [7]:

$$
\hat{U}(\alpha) \cdot \phi(q):=\left(e^{i \alpha q / \hbar} \phi\right)(q)
$$

and,

$$
\hat{V}(\beta) \cdot \phi(q):=e^{\frac{\beta}{d^{2}}(q-\beta / 2)} \phi(q-\beta)
$$

The Hilbert space structure is introduced by the definition of an algebraic state (a positive linear functional) $\omega_{d}: \mathcal{W} \rightarrow \mathbb{C}$, that must coincide with the expectation value in the Hilbert space taken on a special state refered to as the vacuum: $\omega_{d}(a)=\langle\hat{a}\rangle_{\text {vac }}$, for all $a \in \mathcal{W}$. In our case this specification of $J$ induces such a unique state $\omega_{d}$ that yields,

$$
\langle\hat{U}(\alpha)\rangle_{\mathrm{vac}}=e^{-\frac{1}{4} \frac{d^{2} \alpha^{2}}{\hbar^{2}}}
$$

and

$$
\langle\hat{V}(\beta)\rangle_{\mathrm{vac}}=e^{-\frac{1}{4} \frac{\beta^{2}}{d^{2}}}
$$

Note that the exponents in the vacuum expectation values correspond to the metric constructed out of $J$ : $\frac{d^{2} \alpha^{2}}{\hbar^{2}}=g_{d}((0, \alpha) ;(0, \alpha))$ and $\frac{\beta^{2}}{d^{2}}=g_{d}((\beta, 0) ;(\beta, 0))$. Wave functions belong to the space $L^{2}\left(\mathbb{R}, \mathrm{d} \mu_{d}\right)$, where the measure that dictates the inner product in this representation is given by,

$$
\mathrm{d} \mu_{d}=\frac{1}{d \sqrt{\pi}} e^{-\frac{q^{2}}{d^{2}}} \mathrm{~d} q
$$

In this representation, the vacuum is given by the identity function $\phi_{0}(q)=1$ that is, just as any plane wave, normalized. Note that for each value of $d>0$, the representation is well defined and continuous in $\alpha$ and $\beta$. Note also that there is an equivalence between the $q$ representation defined by $d$ and the $k$-representation defined by $1 / d$.

How can we recover then the standard representation in which the measure is given by the Lebesgue measure and the operators are represented as in (4)? It is easy to see that there is an isometric isomorphism $K$ that maps the $d$-representation in $\mathcal{H}_{d}$ to the standard Schrödinger representation in $\mathcal{H}_{\text {schr }}$ by:

$$
\psi(q)=K \cdot \phi(q)=\frac{e^{-\frac{q^{2}}{2 d^{2}}}}{d^{1 / 2} \pi^{1 / 4}} \phi(q) \in \mathcal{H}_{\mathrm{schr}}=L^{2}(\mathbb{R}, \mathrm{d} q)
$$

Thus we see that all $d$-representations are unitarily equivalent. This was to be expected in view of the Stone-Von Neumann uniqueness result. Note also that the vacuum now becomes

$$
\psi_{0}(q)=\frac{1}{d^{1 / 2} \pi^{1 / 4}} e^{-\frac{q^{2}}{2 d^{2}}},
$$

so even when there is no information about the parameter $d$ in the representation itself, it is contained in the vacuum state. This procedure for constructing the GNSSchrödinger representation for quantum mechanics has also been generalized to scalar fields on arbitrary curved space in [8]. Note, however that so far the treatment has all been kinematical, without any knowledge of a Hamiltonian. For the Simple Harmonic Oscillator of mass $m$ and frequency $\omega$, there is a natural choice compatible with the dynamics given by $d=\sqrt{\frac{\hbar}{m \omega}}$, in which some calculations simplify (for instance for coherent states), but in principle one can use any value of $d$. 
Our study will be simplified by focusing on the fundamental entities in the Hilbert Space $\mathcal{H}_{d}$, namely those states generated by acting with $\hat{U}(\alpha)$ on the vacuum $\phi_{0}(q)=1$. Let us denote those states by,

$$
\phi_{\alpha}(q)=\hat{U}(\alpha) \cdot \phi_{0}(q)=e^{i \frac{1}{\hbar} \alpha q}
$$

The inner product between two such states is given by

$$
\left\langle\phi_{\alpha}, \phi_{\lambda}\right\rangle_{d}=\int \mathrm{d} \mu_{d} e^{-\frac{i \alpha q}{\hbar}} e^{\frac{i \lambda q}{\hbar}}=e^{-\frac{(\lambda-\alpha)^{2} d^{2}}{4 \hbar^{2}}}
$$

Note incidentally that, contrary to some common belief, the 'plane waves' in this GNS Hilbert space are indeed normalizable.

Let us now consider the polymer representation. For that, it is important to note that there are two possible limiting cases for the parameter $d$ : i) The limit $1 / d \mapsto 0$ and ii) The case $d \mapsto 0$. In both cases, we have expressions that become ill defined in the representation or measure, so one needs to be careful.

\section{A. The $1 / d \mapsto 0$ case.}

The first observation is that from the expressions (5) and (6) for the algebraic state $\omega_{d}$, we see that the limiting cases are indeed well defined. In our case we get, $\omega_{\mathrm{A}}:=$ $\lim _{1 / d \rightarrow 0} \omega_{d}$ such that,

$$
\omega_{\mathrm{A}}(\hat{U}(\alpha))=\delta_{\alpha, 0} \quad \text { and } \quad \omega_{\mathrm{A}}(\hat{V}(\beta))=1
$$

From this, we can indeed construct the representation by means of the GNS construction. In order to do that and to show how this is obtained we shall consider several expressions. One has to be careful though, since the limit has to be taken with care. Let us consider the measure on the representation that behaves as:

$$
\mathrm{d} \mu_{d}=\frac{1}{d \sqrt{\pi}} e^{-\frac{q^{2}}{d^{2}}} \mathrm{~d} q \mapsto \frac{1}{d \sqrt{\pi}} \mathrm{d} q
$$

so the measures tends to an homogeneous measure but whose 'normalization constant' goes to zero, so the limit becomes somewhat subtle. We shall return to this point later.

Let us now see what happens to the inner product between the fundamental entities in the Hilbert Space $\mathcal{H}_{d}$ given by (7). It is immediate to see that in the $1 / d \mapsto 0$ limit the inner product becomes,

$$
\left\langle\phi_{\alpha}, \phi_{\lambda}\right\rangle_{d} \mapsto \delta_{\alpha, \lambda}
$$

with $\delta_{\alpha, \lambda}$ being Kronecker's delta. We see then that the plane waves $\phi_{\alpha}(q)$ become an orthonormal basis for the new Hilbert space. Therefore, there is a delicate interplay between the two terms that contribute to the measure in order to maintain the normalizability of these functions; we need the measure to become damped (by $1 / d$ ) in order to avoid that the plane waves acquire an infinite norm (as happens with the standard Lebesgue measure), but on the other hand the measure, that for any finite value of $d$ is a Gaussian, becomes more and more spread.

It is important to note that, in this limit, the operators $\hat{U}(\alpha)$ become discontinuous with respect to $\alpha$, given that for any given $\alpha_{1}$ and $\alpha_{2}$ (different), its action on a given basis vector $\psi_{\lambda}(q)$ yields orthogonal vectors. Since the continuity of these operators is one of the hypotesis of the Stone-Von Neumann theorem, the uniqueness result does not apply here. The representation is inequivalent to the standard one.

Let us now analyze the other operator, namely the action of the operator $\hat{V}(\beta)$ on the basis $\phi_{\alpha}(q)$ :

$$
\hat{V}(\beta) \cdot \phi_{\alpha}(q)=e^{-\frac{\beta^{2}}{2 d^{2}}-i \frac{\alpha \beta}{\hbar}} e^{\left(\beta / d^{2}+i \alpha / \hbar\right) q}
$$

which in the limit $1 / d \mapsto 0$ goes to,

$$
\hat{V}(\beta) \cdot \phi_{\alpha}(q) \mapsto e^{i \frac{\alpha \beta}{\hbar}} \phi_{\alpha}(q)
$$

that is continuous on $\beta$. Thus, in the limit, the operator $\hat{p}=-i \hbar \partial_{q}$ is well defined. Also, note that in this limit the operator $\hat{p}$ has $\phi_{\alpha}(q)$ as its eigenstate with eigenvalue given by $\alpha$ :

$$
\hat{p} \cdot \phi_{\alpha}(q) \mapsto \alpha \phi_{\alpha}(q)
$$

To summarize, the resulting theory obtained by taking the limit $1 / d \mapsto 0$ of the ordinary Schrödinger description, that we shall call the 'polymer representation of type A', has the following features: the operators $U(\alpha)$ are well defined but not continuous in $\alpha$, so there is no generator (no operator associated to $q$ ). The basis vectors $\phi_{\alpha}$ are orthonormal (for $\alpha$ taking values on a continuous set) and are eigenvectors of the operator $\hat{p}$ that is well defined. The resulting Hilbert space $\mathcal{H}_{A}$ will be the (A-version of the) polymer representation. Let us now consider the other case, namely, the limit when $d \mapsto 0$.

\section{B. The $d \mapsto 0$ case}

Let us now explore the other limiting case of the Schrödinger/Fock representations labelled by the parameter $d$. Just as in the previous case, the limiting algebraic state becomes, $\omega_{\mathrm{B}}:=\lim _{d \rightarrow 0} \omega_{d}$ such that,

$$
\omega_{\mathrm{B}}(\hat{U}(\alpha))=1 \quad \text { and } \quad \omega_{\mathrm{B}}(\hat{V}(\beta))=\delta_{\beta, 0}
$$

From this positive linear function, one can indeed construct the representation using the GNS construction.

First let us note that the measure, even when the limit has to be taken with due care, behaves as:

$$
\mathrm{d} \mu_{d}=\frac{1}{d \sqrt{\pi}} e^{-\frac{q^{2}}{d^{2}}} \mathrm{~d} q \mapsto \delta(q) \mathrm{d} q
$$

That is, as Dirac's delta distribution. It is immediate to see that, in the $d \mapsto 0$ limit, the inner product between the fundamental states $\phi_{\alpha}(q)$ becomes,

$$
\left\langle\phi_{\alpha}, \phi_{\lambda}\right\rangle_{d} \mapsto 1
$$


This in fact means that the vector $\xi=\phi_{\alpha}-\phi_{\lambda}$ belongs to the Kernel of the limiting inner product, so one has to mod out by these (and all) zero norm states in order to get the Hilbert space.

Let us now analyze the other operator, namely the action of the operator $\hat{V}(\beta)$ on the vacuum $\phi_{0}(q)=1$, which for arbitrary $d$ has the form,

$$
\tilde{\phi}_{\beta}:=\hat{V}(\beta) \cdot \phi_{0}(q)=e^{\frac{\beta}{d^{2}}(q-\beta / 2)}
$$

The inner product between two such states is given by

$$
\left\langle\tilde{\phi}_{\alpha}, \tilde{\phi}_{\beta}\right\rangle_{d}=e^{-\frac{1}{4 d^{2}}(\alpha-\beta)^{2}}
$$

In the limit $d \rightarrow 0,\left\langle\tilde{\phi}_{\alpha}, \tilde{\phi}_{\beta}\right\rangle_{d} \rightarrow \delta_{\alpha, \beta}$. We can see then that it is these functions that become the orthonormal, 'discrete basis' in the theory. However, the function $\tilde{\phi}_{\beta}(q)$ in this limit becomes ill defined. For example, for $\beta>0$, it grows unboundedly for $q>\beta / 2$, is equal to one if $q=\beta / 2$ and zero otherwise. In order to overcome these difficulties and make more transparent the resulting theory, we shall consider the other form of the representation in which the measure is incorporated into the states (and the resulting Hilbert space is $\left.L^{2}(\mathbb{R}, \mathrm{d} q)\right)$. Thus the new state

$$
\begin{aligned}
\psi_{\beta}(q) & :=K \cdot\left(\hat{V}(\beta) \cdot \phi_{0}(q)\right)= \\
& =\frac{1}{(d \sqrt{\pi})^{\frac{1}{2}}} e^{-\frac{1}{2 d^{2}}(q-\beta)^{2}}
\end{aligned}
$$

We can now take the limit and what we get is

$$
\lim _{d \mapsto 0} \psi_{\beta}(q):=\delta^{1 / 2}(q, \beta)
$$

where by $\delta^{1 / 2}(q, \beta)$ we mean something like 'the square root of the Dirac distribution'. What we really mean is an object that satisfies the following property:

$$
\delta^{1 / 2}(q, \beta) \cdot \delta^{1 / 2}(q, \alpha)=\delta(q, \beta) \delta_{\beta, \alpha}
$$

That is, if $\alpha=\beta$ then it is just the ordinary delta, otherwise it is zero. In a sense these object can be regarded as half-densities that can not be integrated by themselves, but whose product can. We conclude then that the inner product is,

$$
\left\langle\psi_{\beta}, \psi_{\alpha}\right\rangle=\int_{\mathbb{R}} \mathrm{d} q \overline{\psi_{\beta}}(q) \psi_{\alpha}(q)=\int_{\mathbb{R}} \mathrm{d} q \delta(q, \alpha) \delta_{\beta, \alpha}=\delta_{\beta, \alpha}
$$

which is just what we expected. Note that in this representation, the vacuum state becomes $\psi_{0}(q):=\delta^{1 / 2}(q, 0)$, namely, the half-delta with support in the origin. It is important to note that we are arriving in a natural way to states as half-densities, whose squares can be integrated without the need of a nontrivial measure on the configuration space. Diffeomorphism invariance arises then in a natural but subtle manner.

Note that as the end result we recover the Kronecker delta inner product for the new fundamental states:

$$
\chi_{\beta}(q):=\delta^{1 / 2}(q, \beta) .
$$

Thus, in this new B-polymer representation, the Hilbert space $\mathcal{H}_{\mathrm{B}}$ is the completion with respect to the inner product (13) of the states generated by taking (finite) linear combinations of basis elements of the form $\chi_{\beta}$ :

$$
\Psi(q)=\sum_{i} b_{i} \chi_{\beta_{i}}(q)
$$

Let us now introduce an equivalent description of this Hilbert space. Instead of having the basis elements be half-deltas as elements of the Hilbert space where the inner product is given by the ordinary Lebesgue measure $\mathrm{d} q$, we redefine both the basis and the measure. We could consider, instead of a half-delta with support $\beta$, a Kronecker delta or characteristic function with support on $\beta$ :

$$
\chi_{\beta}^{\prime}(q):=\delta_{q, \beta}
$$

These functions have a similar behavior with respect to the product as the half-deltas, namely: $\chi_{\beta}^{\prime}(q) \cdot \chi_{\alpha}^{\prime}(q)=$ $\delta_{\beta, \alpha}$. The main difference is that neither $\chi^{\prime}$ nor their squares are integrable with respect to the Lebesgue measure (having zero norm). In order to fix that problem we have to change the measure so that we recover the basic inner product (13) with our new basis. The needed measure turns out to be the discrete counting measure on $\mathbb{R}$. Thus any state in the 'half density basis' can be written (using the same expression) in terms of the 'Kronecker basis'. For more details and further motivation see the next section.

Note that in this B-polymer representation, both $\hat{U}$ and $\hat{V}$ have their roles interchanged with that of the A-polymer representation: while $U(\alpha)$ is discontinuous and thus $\hat{q}$ is not defined in the A-representation, we have that it is $V(\beta)$ in the B-representation that has this property. In this case, it is the operator $\hat{p}$ that can not be defined. We see then that given a physical system for which the configuration space has a well defined physical meaning, within the possible representation in which wave-functions are functions of the configuration variable $q$, the $\mathrm{A}$ and $\mathrm{B}$ polymer representations are radically different and inequivalent.

Having said this, it is also true that the A and B representations are equivalent in a different sense, by means of the duality between $q$ and $p$ representations and the $d \leftrightarrow 1 / d$ duality: The A-polymer representation in the "q-representation" is equivalent to the B-polymer representation in the " $p$-representation", and conversely. When studying a problem, it is important to decide from the beginning which polymer representation (if any) one should be using (for instance in the $q$-polarization). This has as a consequence an implication on which variable is naturally "quantized" (even if continuous): $p$ for A and $q$ for B. There could be for instance a physical criteria for this choice. For example a fundamental symmetry could suggest that one representation is more natural than another one. This indeed has been recently noted by Chiou in [10], where the Galileo group is investigated and where it is shown that the $\mathrm{B}$ representation is better behaved. 
In the other polarization, namely for wavefunctions of $p$, the picture gets reversed: $q$ is discrete for the Arepresentation, while $p$ is for the B-case. Let us end this section by noting that the procedure of obtaining the polymer quantization by means of an appropriate limit of Fock-Schrödinger representations might prove useful in more general settings in field theory or quantum gravity.

\section{POLYMER QUANTUM MECHANICS: KINEMATICS}

In previous sections we have derived what we have called the $\mathrm{A}$ and $\mathrm{B}$ polymer representations (in the $q$ polarization) as limiting cases of ordinary Fock representations. In this section, we shall describe, without any reference to the Schrödinger representation, the 'abstract' polymer representation and then make contact with its two possible realizations, closely related to the A and $B$ cases studied before. What we will see is that one of them (the A case) will correspond to the $p$-polarization while the other one corresponds to the $q$-representation, when a choice is made about the physical significance of the variables.

We can start by defining abstract kets $|\mu\rangle$ labelled by a real number $\mu$. These shall belong to the Hilbert space $\mathcal{H}_{\text {poly. }}$. From these states, we define a generic 'cylinder states' that correspond to a choice of a finite collection of numbers $\mu_{i} \in \mathbb{R}$ with $i=1,2, \ldots, N$. Associated to this choice, there are $N$ vectors $\left|\mu_{i}\right\rangle$, so we can take a linear combination of them

$$
|\psi\rangle=\sum_{i=1}^{N} a_{i}\left|\mu_{i}\right\rangle
$$

The polymer inner product between the fundamental kets is given by,

$$
\langle\nu \mid \mu\rangle=\delta_{\nu, \mu}
$$

That is, the kets are orthogonal to each other (when $\nu \neq$ $\mu)$ and they are normalized $(\langle\mu \mid \mu\rangle=1)$. Immediately, this implies that, given any two vectors $|\phi\rangle=\sum_{j=1}^{M} b_{j}\left|\nu_{j}\right\rangle$ and $|\psi\rangle=\sum_{i=1}^{N} a_{i}\left|\mu_{i}\right\rangle$, the inner product between them is given by,

$$
\langle\phi \mid \psi\rangle=\sum_{i} \sum_{j} \bar{b}_{j} a_{i}\left\langle\nu_{j} \mid \mu_{i}\right\rangle=\sum_{k} \bar{b}_{k} a_{k}
$$

where the sum is over $k$ that labels the intersection points between the set of labels $\left\{\nu_{j}\right\}$ and $\left\{\mu_{i}\right\}$. The Hilbert space $\mathcal{H}_{\text {poly }}$ is the Cauchy completion of finite linear combination of the form (15) with respect to the inner product (16). $\mathcal{H}_{\text {poly }}$ is non-separable. There are two basic operators on this Hilbert space: the 'label operator' $\hat{\varepsilon}$ :

$$
\hat{\varepsilon}|\mu\rangle:=\mu|\mu\rangle
$$

and the displacement operator $\hat{\mathbf{s}}(\lambda)$,

$$
\hat{\mathbf{s}}(\lambda)|\mu\rangle:=|\mu+\lambda\rangle
$$

The operator $\hat{\varepsilon}$ is symmetric and the operator $(\mathrm{s}) \hat{\mathbf{s}}(\lambda)$ defines a one-parameter family of unitary operators on $\mathcal{H}_{\text {poly }}$, where its adjoint is given by $\hat{\mathbf{s}}^{\dagger}(\lambda)=\hat{\mathbf{s}}(-\lambda)$. This action is however, discontinuous with respect to $\lambda$ given that $|\mu\rangle$ and $|\mu+\lambda\rangle$ are always orthogonal, no matter how small is $\lambda$. Thus, there is no (Hermitian) operator that could generate $\hat{\mathbf{s}}(\lambda)$ by exponentiation.

So far we have given the abstract characterization of the Hilbert space, but one would like to make contact with concrete realizations as wave functions, or by identifying the abstract operators $\hat{\varepsilon}$ and $\hat{\mathbf{s}}$ with physical operators.

Suppose we have a system with a configuration space with coordinate given by $q$, and $p$ denotes its canonical conjugate momenta. Suppose also that for physical reasons we decide that the configuration coordinate $q$ will have some "discrete character" (for instance, if it is to be identified with position, one could say that there is an underlying discreteness in position at a small scale). How can we implement such requirements by means of the polymer representation? There are two possibilities, depending on the choice of 'polarizations' for the wavefunctions, namely whether they will be functions of configuration $q$ or momenta $p$. Let us the divide the discussion into two parts.

\section{A. Momentum polarization}

In this polarization, states will be denoted by,

$$
\psi(p)=\langle p \mid \psi\rangle
$$

where

$$
\psi_{\mu}(p)=\langle p \mid \mu\rangle=e^{i \frac{\mu p}{\hbar}}
$$

How are then the operators $\hat{\varepsilon}$ and $\hat{\mathbf{s}}$ represented? Note that if we associate the multiplicative operator

$$
\hat{V}(\lambda) \cdot \psi_{\mu}(p)=e^{i \frac{\lambda p}{\hbar}} e^{i \frac{\mu p}{\hbar}}=e^{i \frac{(\mu+\lambda)}{\hbar} p}=\psi_{(\mu+\lambda)}(p)
$$

we see then that the operator $\hat{V}(\lambda)$ corresponds precisely to the shift operator $\hat{\mathbf{s}}(\lambda)$. Thus we can also conclude that the operator $\hat{p}$ does not exist. It is now easy to identify the operator $\hat{q}$ with:

$$
\hat{q} \cdot \psi_{\mu}(p)=-i \hbar \frac{\partial}{\partial p} \psi_{\mu}(p)=\mu e^{i \frac{\mu p}{\hbar}}=\mu \psi_{\mu}(p)
$$

namely, with the abstract operator $\hat{\varepsilon}$. The reason we say that $\hat{q}$ is discrete is because this operator has as its eigenvalue the label $\mu$ of the elementary state $\psi_{\mu}(p)$, and this label, even when it can take value in a continuum of possible values, is to be understood as a discrete set, given that the states are orthonormal for all values of $\mu$. Given that states are now functions of $p$, the inner product (16) should be defined by a measure $\mu$ on the space on which the wave-functions are defined. In order 
to know what these two objects are, namely, the quantum "configuration" space $\mathcal{C}$ and the measure thereon ${ }^{1}$, we have to make use of the tools available to us from the theory of $C^{*}$-algebras. If we consider the operators $\hat{V}(\lambda)$, together with their natural product and $*$-relation given by $\hat{V}^{*}(\lambda)=\hat{V}(-\lambda)$, they have the structure of an Abelian $C^{*}$-algebra (with unit) $\mathcal{A}$. We know from the representation theory of such objects that $\mathcal{A}$ is isomorphic to the space of continuous functions $C^{0}(\Delta)$ on a compact space $\Delta$, the spectrum of $\mathcal{A}$. Any representation of $\mathcal{A}$ on a Hilbert space as multiplication operator will be on spaces of the form $L^{2}(\Delta, \mathrm{d} \mu)$. That is, our quantum configuration space is the spectrum of the algebra, which in our case corresponds to the Bohr compactification $\mathbb{R}_{\mathrm{b}}$ of the real line [11]. This space is a compact group and there is a natural probability measure defined on it, the Haar measure $\mu_{\mathrm{H}}$. Thus, our Hilbert space $\mathcal{H}_{\text {poly }}$ will be isomorphic to the space,

$$
\mathcal{H}_{\text {poly }, p}=L^{2}\left(\mathbb{R}_{\mathrm{b}}, \mathrm{d} \mu_{\mathrm{H}}\right)
$$

In terms of 'quasi periodic functions' generated by $\psi_{\mu}(p)$, the inner product takes the form

$$
\begin{aligned}
\left\langle\psi_{\mu} \mid \psi_{\lambda}\right\rangle & :=\int_{\mathbb{R}_{\mathrm{b}}} \mathrm{d} \mu_{\mathrm{H}} \overline{\psi_{\mu}}(p) \psi_{\lambda}(p):= \\
& =\lim _{L \mapsto \infty} \frac{1}{2 L} \int_{-L}^{L} \mathrm{~d} p \overline{\psi_{\mu}}(p) \psi_{\lambda}(p)=\delta_{\mu, \lambda}
\end{aligned}
$$

note that in the $p$-polarization, this characterization corresponds to the 'A-version' of the polymer representation of Sec. II (where $p$ and $q$ are interchanged).

\section{B. q-polarization}

Let us now consider the other polarization in which wave functions will depend on the configuration coordinate $q$ :

$$
\psi(q)=\langle q \mid \psi\rangle
$$

The basic functions, that now will be called $\tilde{\psi}_{\mu}(q)$, should be, in a sense, the dual of the functions $\psi_{\mu}(p)$ of the previous subsection. We can try to define them via a 'Fourier transform':

$$
\tilde{\psi}_{\mu}(q):=\langle q \mid \mu\rangle=\left\langle q\left|\int_{\mathbb{R}_{\mathrm{b}}} \mathrm{d} \mu_{\mathrm{H}}\right| p\right\rangle\langle p \mid \mu\rangle
$$

which is given by

$$
\begin{aligned}
\tilde{\psi}_{\mu}(q) & :=\int_{\mathbb{R}_{\mathrm{b}}} \mathrm{d} \mu_{\mathrm{H}}\langle q \mid p\rangle \psi_{\mu}(p)= \\
& =\int_{\mathbb{R}_{\mathrm{b}}} \mathrm{d} \mu_{\mathrm{H}} e^{-i \frac{p q}{\hbar}} e^{i \frac{\mu p}{\hbar}}=\delta_{q, \mu}
\end{aligned}
$$

\footnotetext{
1 here we use the standard terminology of 'configuration space' to denote the domain of the wave function even when, in this case, it corresponds to the physical momenta $p$.
}

That is, the basic objects in this representation are Kronecker deltas. This is precisely what we had found in Sec. II for the B-type representation. How are now the basic operators represented and what is the form of the inner product? Regarding the operators, we expect that they are represented in the opposite manner as in the previous $p$-polarization case, but that they preserve the same features: $\hat{p}$ does not exist (the derivative of the Kronecker delta is ill defined), but its exponentiated version $\hat{V}(\lambda)$ does:

$$
\hat{V}(\lambda) \cdot \psi(q)=\psi(q+\lambda)
$$

and the operator $\hat{q}$ that now acts as multiplication has as its eigenstates, the functions $\tilde{\psi}_{\nu}(q)=\delta_{\nu, q}$ :

$$
\hat{q} \cdot \tilde{\psi}_{\mu}(q):=\mu \tilde{\psi}_{\mu}(q)
$$

What is now the nature of the quantum configurations space $\mathcal{Q}$ ? And what is the measure thereon $\mathrm{d} \mu_{q}$ ? that defines the inner product we should have:

$$
\left\langle\tilde{\psi}_{\mu}(q), \tilde{\psi}_{\lambda}(q)\right\rangle=\delta_{\mu, \lambda}
$$

The answer comes from one of the characterizations of the Bohr compactification: we know that it is, in a precise sense, dual to the real line but when equipped with the discrete topology $\mathbb{R}_{\mathrm{d}}$. Furthermore, the measure on $\mathbb{R}_{\mathrm{d}}$ will be the 'counting measure'. In this way we recover the same properties we had for the previous characterization of the polymer Hilbert space. We can thus write:

$$
\mathcal{H}_{\text {poly }, x}:=L^{2}\left(\mathbb{R}_{\mathrm{d}}, \mathrm{d} \mu_{\mathrm{c}}\right)
$$

This completes a precise construction of the B-type polymer representation sketched in the previous section. Note that if we had chosen the opposite physical situation, namely that $q$, the configuration observable, be the quantity that does not have a corresponding operator, then we would have had the opposite realization: In the $q$ polarization we would have had the type-A polymer representation and the type-B for the $p$-polarization. As we shall see both scenarios have been considered in the literature.

Up to now we have only focused our discussion on the kinematical aspects of the quantization process. Let us now consider in the following section the issue of dynamics and recall the approach that had been adopted in the literature, before the issue of the removal of the regulator was reexamined in [6].

\section{POLYMER QUANTUM MECHANICS: DYNAMICS}

As we have seen the construction of the polymer representation is rather natural and leads to a quantum theory with different properties than the usual Schrödinger counterpart such as its non-separability, the 
non-existence of certain operators and the existence of normalized eigen-vectors that yield a precise value for one of the phase space coordinates. This has been done without any regard for a Hamiltonian that endows the system with a dynamics, energy and so on.

First let us consider the simplest case of a particle of mass $m$ in a potential $V(q)$, in which the Hamiltonian $H$ takes the form,

$$
H=\frac{1}{2 m} p^{2}+V(q)
$$

Suppose furthermore that the potential is given by a nonperiodic function, such as a polynomial or a rational function. We can immediately see that a direct implementation of the Hamiltonian is out of our reach, for the simple reason that, as we have seen, in the polymer representation we can either represent $q$ or $p$, but not both! What has been done so far in the literature? The simplest thing possible: approximate the non-existing term by a well defined function that can be quantized and hope for the best. As we shall see in next sections, there is indeed more that one can do.

At this point there is also an important decision to be made: which variable $q$ or $p$ should be regarded as "discrete"? Once this choice is made, then it implies that the other variable will not exist: if $q$ is regarded as discrete, then $p$ will not exist and we need to approximate the kinetic term $p^{2} / 2 m$ by something else; if $p$ is to be the discrete quantity, then $q$ will not be defined and then we need to approximate the potential $V(q)$. What happens with a periodic potential? In this case one would be modelling, for instance, a particle on a regular lattice such as a phonon living on a crystal, and then the natural choice is to have $q$ not well defined. Furthermore, the potential will be well defined and there is no approximation needed.

In the literature both scenarios have been considered. For instance, when considering a quantum mechanical system in [2], the position was chosen to be discrete, so $p$ does not exist, and one is then in the A type for the momentum polarization (or the type B for the $q$ polarization). With this choice, it is the kinetic term the one that has to be approximated, so once one has done this, then it is immediate to consider any potential that will thus be well defined. On the other hand, when considering loop quantum cosmology (LQC), the standard choice is that the configuration variable is not defined [4]. This choice is made given that LQC is regarded as the symmetric sector of full loop quantum gravity where the connection (that is regarded as the configuration variable) can not be promoted to an operator and one can only define its exponentiated version, namely, the holonomy. In that case, the canonically conjugate variable, closely related to the volume, becomes 'discrete', just as in the full theory. This case is however, different from the particle in a potential example. First we could mention that the functional form of the Hamiltonian constraint that implements dynamics has a different structure, but the more important difference lies in that the system is constrained.

Let us return to the case of the particle in a potential and for definiteness, let us start with the auxiliary kinematical framework in which: $q$ is discrete, $p$ can not be promoted and thus we have to approximate the kinetic term $\hat{p}^{2} / 2 m$. How is this done? The standard prescription is to define, on the configuration space $\mathcal{C}$, a regular 'graph' $\gamma_{\mu_{0}}$. This consists of a numerable set of points, equidistant, and characterized by a parameter $\mu_{0}$ that is the (constant) separation between points. The simplest example would be to consider the set $\gamma_{\mu_{0}}=\left\{q \in \mathbb{R} \mid q=n \mu_{0}, \forall n \in \mathbb{Z}\right\}$.

This means that the basic kets that will be considered $\left|\mu_{n}\right\rangle$ will correspond precisely to labels $\mu_{n}$ belonging to the graph $\gamma_{\mu_{0}}$, that is, $\mu_{n}=n \mu_{0}$. Thus, we shall only consider states of the form,

$$
|\psi\rangle=\sum_{n} b_{n}\left|\mu_{n}\right\rangle
$$

This 'small' Hilbert space $\mathcal{H}_{\gamma_{\mu_{0}}}$, the graph Hilbert space, is a subspace of the 'large' polymer Hilbert space $\mathcal{H}_{\text {poly }}$ but it is separable. The condition for a state of the form (21) to belong to the Hilbert space $\mathcal{H}_{\gamma_{\mu_{0}}}$ is that the coefficients $b_{n}$ satisfy: $\sum_{n}\left|b_{n}\right|^{2}<\infty$.

Let us now consider the kinetic term $\hat{p}^{2} / 2 m$. We have to approximate it by means of trigonometric functions, that can be built out of the functions of the form $e^{i \lambda p / \hbar}$. As we have seen in previous sections, these functions can indeed be promoted to operators and act as translation operators on the kets $|\mu\rangle$. If we want to remain in the graph $\gamma$, and not create 'new points', then one is constrained to considering operators that displace the kets by just the right amount. That is, we want the basic shift operator $\hat{V}(\lambda)$ to be such that it maps the ket with label $\left|\mu_{n}\right\rangle$ to the next ket, namely $\left|\mu_{n+1}\right\rangle$. This can indeed achieved by fixing, once and for all, the value of the allowed parameter $\lambda$ to be $\lambda=\mu_{0}$. We have then,

$$
\hat{V}\left(\mu_{0}\right) \cdot\left|\mu_{n}\right\rangle=\left|\mu_{n}+\mu_{0}\right\rangle=\left|\mu_{n+1}\right\rangle
$$

which is what we wanted. This basic 'shift operator' will be the building block for approximating any (polynomial) function of $p$. In order to do that we notice that the function $p$ can be approximated by,

$$
p \approx \frac{\hbar}{\mu_{0}} \sin \left(\frac{\mu_{0} p}{\hbar}\right)=\frac{\hbar}{2 i \mu_{0}}\left(e^{i \frac{\mu_{0} p}{\hbar}}-e^{-i \frac{\mu_{0} p}{\hbar}}\right)
$$

where the approximation is good for $p<<\hbar / \mu_{0}$. Thus, one can define a regulated operator $\hat{p}_{\mu_{0}}$ that depends on the 'scale' $\mu_{0}$ as:

$$
\begin{aligned}
\hat{p}_{\mu_{0}} \cdot\left|\mu_{n}\right\rangle & :=\frac{\hbar}{2 i \mu_{0}}\left[V\left(\mu_{0}\right)-V\left(-\mu_{0}\right)\right] \cdot\left|\mu_{n}\right\rangle= \\
& =\frac{i \hbar}{2 \mu_{0}}\left(\left|\mu_{n+1}\right\rangle-\left|\mu_{n-1}\right\rangle\right)
\end{aligned}
$$

In order to regulate the operator $\hat{p}^{2}$, there are (at least) two possibilities, namely to compose the operator $\hat{p}_{\mu_{0}}$ 
with itself or to define a new approximation. The operator $\hat{p}_{\mu_{0}} \cdot \hat{p}_{\mu_{0}}$ has the feature that shifts the states two steps in the graph to both sides. There is however another operator that only involves shifting once:

$$
\begin{aligned}
\hat{p}_{\mu_{0}}^{2} \cdot\left|\nu_{n}\right\rangle & :=\frac{\hbar^{2}}{\mu_{0}^{2}}\left[2-\hat{V}\left(\mu_{0}\right)-\hat{V}\left(-\mu_{0}\right)\right] \cdot\left|\nu_{n}\right\rangle= \\
& =\frac{\hbar^{2}}{\mu_{0}^{2}}\left(2\left|\nu_{n}\right\rangle-\left|\nu_{n+1}\right\rangle-\left|\nu_{n-1}\right\rangle\right)
\end{aligned}
$$

which corresponds to the approximation $p^{2} \approx \frac{2 \hbar^{2}}{\mu_{0}^{2}}(1-$ $\left.\cos \left(\mu_{0} p / \hbar\right)\right)$, valid also in the regime $p<<\hbar / \mu_{0}$. With these considerations, one can define the operator $\hat{H}_{\mu_{0}}$, the Hamiltonian at scale $\mu_{0}$, that in practice 'lives' on the space $\mathcal{H}_{\gamma_{\mu_{0}}}$ as,

$$
\hat{H}_{\mu_{0}}:=\frac{1}{2 m} \hat{p}_{\mu_{0}}^{2}+\hat{V}(q)
$$

that is a well defined, symmetric operator on $\mathcal{H}_{\gamma_{\mu_{0}}}$. Notice that the operator is also defined on $\mathcal{H}_{\text {poly }}$, but there its physical interpretation is problematic. For example, it turns out that the expectation value of the kinetic term calculated on most states (states which are not tailored to the exact value of the parameter $\mu_{0}$ ) is zero. Even if one takes a state that gives "reasonable" expectation values of the $\mu_{0}$-kinetic term and uses it to calculate the expectation value of the kinetic term corresponding to a slight perturbation of the parameter $\mu_{0}$ one would get zero. This problem, and others that arise when working on $\mathcal{H}_{\text {poly }}$, forces one to assign a physical interpretation to the Hamiltonian $\hat{H}_{\mu_{0}}$ only when its action is restricted to the subspace $\mathcal{H}_{\gamma_{\mu_{0}}}$.

Let us now explore the form that the Hamiltonian takes in the two possible polarizations. In the $q$-polarization, the basis, labelled by $n$ is given by the functions $\chi_{n}(q)=$ $\delta_{q, \mu_{n}}$. That is, the wave functions will only have support on the set $\gamma_{\mu_{0}}$. Alternatively, one can think of a state as completely characterized by the 'Fourier coefficients' $a_{n}: \psi(q) \leftrightarrow a_{n}$, which is the value that the wave function $\psi(q)$ takes at the point $q=\mu_{n}=n \mu_{0}$. Thus, the Hamiltonian takes the form of a difference equation when acting on a general state $\psi(q)$. Solving the time independent Schrödinger equation $\hat{H} \cdot \psi=E \psi$ amounts to solving the difference equation for the coefficients $a_{n}$.

The momentum polarization has a different structure. In this case, the operator $\hat{p}_{\mu_{0}}^{2}$ acts as a multiplication operator,

$$
\hat{p}_{\mu_{0}}^{2} \cdot \psi(p)=\frac{2 \hbar^{2}}{\mu_{0}^{2}}\left[1-\cos \left(\frac{\mu_{0} p}{\hbar}\right)\right] \psi(p)
$$

The operator corresponding to $q$ will be represented as a derivative operator

$$
\hat{q} \cdot \psi(p):=i \hbar \partial_{p} \psi(p)
$$

For a generic potential $V(q)$, it has to be defined by means of spectral theory defined now on a circle. Why on a circle? For the simple reason that by restricting ourselves to a regular graph $\gamma_{\mu_{0}}$, the functions of $p$ that preserve it (when acting as shift operators) are of the form $e^{\left(i m \mu_{0} p / \hbar\right)}$ for $m$ integer. That is, what we have are Fourier modes, labelled by $m$, of period $2 \pi \hbar / \mu_{0}$ in $p$. Can we pretend then that the phase space variable $p$ is now compactified? The answer is in the affirmative. The inner product on periodic functions $\psi_{\mu_{0}}(p)$ of $p$ coming from the full Hilbert space $\mathcal{H}_{\text {poly }}$ and given by

$$
\langle\phi(p) \mid \psi(p)\rangle_{\text {poly }}=\lim _{L \mapsto \infty} \frac{1}{2 L} \int_{-L}^{L} \mathrm{~d} p \bar{\phi}(p) \psi(p)
$$

is precisely equivalent to the inner product on the circle given by the uniform measure

$$
\langle\phi(p) \mid \psi(p)\rangle_{\mu_{0}}=\frac{\mu_{0}}{2 \pi \hbar} \int_{-\pi \hbar / \mu_{0}}^{\pi \hbar / \mu_{0}} \mathrm{~d} p \bar{\phi}(p) \psi(p)
$$

with $p \in\left(-\pi \hbar / \mu_{0}, \pi \hbar / \mu_{0}\right)$. As long as one restricts attention to the graph $\gamma_{\mu_{0}}$, one can work in this separable Hilbert space $\mathcal{H}_{\gamma_{\mu_{0}}}$ of square integrable functions on $S^{1}$. Immediately, one can see the limitations of this description. If the mechanical system to be quantized is such that its orbits have values of the momenta $p$ that are not small compared with $\pi \hbar / \mu_{0}$ then the approximation taken will be very poor, and we don't expect neither the effective classical description nor its quantization to be close to the standard one. If, on the other hand, one is always within the region in which the approximation can be regarded as reliable, then both classical and quantum descriptions should approximate the standard description.

What does 'close to the standard description' exactly mean needs, of course, some further clarification. In particular one is assuming the existence of the usual Schrödinger representation in which the system has a behavior that is also consistent with observations. If this is the case, the natural question is: How can we approximate such description from the polymer picture? Is there a fine enough graph $\gamma_{\mu_{0}}$ that will approximate the system in such a way that all observations are indistinguishable? Or even better, can we define a procedure, that involves a refinement of the graph $\gamma_{\mu_{0}}$ such that one recovers the standard picture?

It could also happen that a continuum limit can be defined but does not coincide with the 'expected one'. But there might be also physical systems for which there is no standard description, or it just does not make sense. Can in those cases the polymer representation, if it exists, provide the correct physical description of the system under consideration? For instance, if there exists a physical limitation to the minimum scale set by $\mu_{0}$, as could be the case for a quantum theory of gravity, then the polymer description would provide a true physical bound on the value of certain quantities, such as $p$ in our example. This could be the case for loop quantum cosmology, where there is a minimum value for physical volume (coming from the full theory), and phase space points near the 'singularity' lie at the region where the 
approximation induced by the scale $\mu_{0}$ departs from the standard classical description. If in that case the polymer quantum system is regarded as more fundamental than the classical system (or its standard Wheeler-De Witt quantization), then one would interpret this discrepancies in the behavior as a signal of the breakdown of classical description (or its 'naive' quantization).

In the next section we present a method to remove the regulator $\mu_{0}$ which was introduced as an intermediate step to construct the dynamics. More precisely, we shall consider the construction of a continuum limit of the polymer description by means of a renormalization procedure.

\section{THE CONTINUUM LIMIT}

This section has two parts. In the first one we motivate the need for a precise notion of the continuum limit of the polymeric representation, explaining why the most direct, and naive approach does not work. In the second part, we shall present the main ideas and results of the paper [6], where the Hamiltonian and the physical Hilbert space in polymer quantum mechanics are constructed as a continuum limit of effective theories, following Wilson's renormalization group ideas. The resulting physical Hilbert space turns out to be unitarily isomorphic to the ordinary $\mathcal{H}_{s}=L^{2}(\mathbb{R}, \mathrm{d} q)$ of the Schrödinger theory.

Before describing the results of [6] we should discuss the precise meaning of reaching a theory in the continuum. Let us for concreteness consider the B-type representation in the $q$-polarization. That is, states are functions of $q$ and the orthonormal basis $\chi_{\mu}(q)$ is given by characteristic functions with support on $q=\mu$. Let us now suppose we have a Schrödinger state $\Psi(q) \in \mathcal{H}_{s}=$ $L^{2}(\mathbb{R}, \mathrm{d} q)$. What is the relation between $\Psi(q)$ and a state in $\mathcal{H}_{\text {poly }, x}$ ? We are also interested in the opposite question, that is, we would like to know if there is a preferred state in $\mathcal{H}_{s}$ that is approximated by an arbitrary state $\psi(q)$ in $\mathcal{H}_{\text {poly }, x}$. The first obvious observation is that a Schödinger state $\Psi(q)$ does not belong to $\mathcal{H}_{\text {poly }, x}$ since it would have an infinite norm. To see that note that even when the would-be state can be formally expanded in the $\chi_{\mu}$ basis as,

$$
\Psi(q)=\sum_{\mu} \Psi(\mu) \chi_{\mu}(q)
$$

where the sum is over the parameter $\mu \in \mathbb{R}$. Its associated norm in $\mathcal{H}_{\text {poly }, x}$ would be:

$$
|\Psi(q)|_{\text {poly }}^{2}=\sum_{\mu}|\Psi(\mu)|^{2} \rightarrow \infty
$$

which blows up. Note that in order to define a mapping $P: \mathcal{H}_{s} \rightarrow \mathcal{H}_{\text {poly }, x}$, there is a huge ambiguity since the values of the function $\Psi(q)$ are needed in order to expand the polymer wave function. Thus we can only define a mapping in a dense subset $\mathcal{D}$ of $\mathcal{H}_{s}$ where the values of the functions are well defined (recall that in $\mathcal{H}_{s}$ the value of functions at a given point has no meaning since states are equivalence classes of functions). We could for instance ask that the mapping be defined for representatives of the equivalence classes in $\mathcal{H}_{s}$ that are piecewise continuous. From now on, when we refer to an element of the space $\mathcal{H}_{s}$ we shall be refereeing to one of those representatives. Notice then that an element of $\mathcal{H}_{s}$ does define an element of $\mathrm{Cyl}_{\gamma}^{*}$, the dual to the space $\mathrm{Cyl}_{\gamma}$, that is, the space of cylinder functions with support on the (finite) lattice $\gamma=\left\{\mu_{1}, \mu_{2}, \ldots, \mu_{N}\right\}$, in the following way:

$$
\Psi(q): \mathrm{Cyl}_{\gamma} \longrightarrow \mathbb{C}
$$

such that

$$
\begin{aligned}
\Psi(q)[\psi(q)] & =\left(\Psi|\psi\rangle:=\sum_{\mu} \overline{\Psi(\mu)}\left\langle\chi_{\mu} \mid \sum_{i=1}^{N} \psi_{i} \chi_{\mu_{i}}\right\rangle_{\text {poly }_{\gamma}}\right. \\
& =\sum_{i=1}^{N} \overline{\Psi\left(\mu_{i}\right)} \psi_{i}<\infty
\end{aligned}
$$

Note that this mapping could be seen as consisting of two parts: First, a projection $P_{\gamma}: \mathrm{Cyl}^{*} \rightarrow \mathrm{Cyl}_{\gamma}$ such that $P_{\gamma}(\Psi)=\Psi_{\gamma}(q):=\sum_{i} \Psi\left(\mu_{i}\right) \chi_{\mu_{i}}(q) \in \mathrm{Cyl}_{\gamma}$. The state $\Psi_{\gamma}$ is sometimes refereed to as the 'shadow of $\Psi(q)$ on the lattice $\gamma$ '. The second step is then to take the inner product between the shadow $\Psi_{\gamma}(q)$ and the state $\psi(q)$ with respect to the polymer inner product $\left\langle\Psi_{\gamma} \mid \psi\right\rangle_{\text {poly }_{\gamma}}$. Now this inner product is well defined. Notice that for any given lattice $\gamma$ the corresponding projector $P_{\gamma}$ can be intuitively interpreted as some kind of 'coarse graining map' from the continuum to the lattice $\gamma$. In terms of functions of $q$ the projection is replacing a continuous function defined on $\mathbb{R}$ with a function over the lattice $\gamma \subset \mathbb{R}$ which is a discrete set simply by restricting $\Psi$ to $\gamma$. The finer the lattice the more points that we have on the curve. As we shall see in the second part of this section, there is indeed a precise notion of coarse graining that implements this intuitive idea in a concrete fashion. In particular, we shall need to replace the lattice $\gamma$ with a decomposition of the real line in intervals (having the lattice points as end points).

Let us now consider a system in the polymer representation in which a particular lattice $\gamma_{0}$ was chosen, say with points of the form $\left\{q_{k} \in \mathbb{R} \mid q_{k}=k a_{0}, \forall k \in \mathbb{Z}\right\}$, namely a uniform lattice with spacing equal to $a_{0}$. In this case, any Schrödinger wave function (of the type that we consider) will have a unique shadow on the lattice $\gamma_{0}$. If we refine the lattice $\gamma \mapsto \gamma_{n}$ by dividing each interval in $2^{n}$ new intervals of length $a_{n}=a_{0} / 2^{n}$ we have new shadows that have more and more points on the curve. Intuitively, by refining infinitely the graph we would recover the original function $\Psi(q)$. Even when at each finite step the corresponding shadow has a finite norm in the polymer Hilbert space, the norm grows unboundedly and the 
limit can not be taken, precisely because we can not embed $\mathcal{H}_{s}$ into $\mathcal{H}_{\text {poly }}$. Suppose now that we are interested in the reverse process, namely starting from a polymer theory on a lattice and asking for the 'continuum wave function' that is best approximated by a wave function over a graph. Suppose furthermore that we want to consider the limit of the graph becoming finer. In order to give precise answers to these (and other) questions we need to introduce some new technology that will allow us to overcome these apparent difficulties. In the remaining of this section we shall recall these constructions for the benefit of the reader. Details can be found in [6] (which is an application of the general formalism discussed in [9]).

The starting point in this construction is the concept of a scale $C$, which allows us to define the effective theories and the concept of continuum limit. In our case a scale is a decomposition of the real line in the union of closed-open intervals, that cover the whole line and do not intersect. Intuitively, we are shifting the emphasis from the lattice points to the intervals defined by the same points with the objective of approximating continuous functions defined on $\mathbb{R}$ with functions that are constant on the intervals defined by the lattice. To be precise, we define an embedding, for each scale $C_{n}$ from $\mathcal{H}_{\text {poly }}$ to $\mathcal{H}_{s}$ by means of a step function:

$\sum_{m} \Psi\left(m a_{n}\right) \chi_{m a_{n}}(q) \rightarrow \sum_{m} \Psi\left(m a_{n}\right) \chi_{\alpha_{m}}(q) \in \mathcal{H}_{s}$

with $\chi_{\alpha_{n}}(q)$ a characteristic function on the interval $\alpha_{m}=\left[m a_{n},(m+1) a_{n}\right)$. Thus, the shadows (living on the lattice) were just an intermediate step in the construction of the approximating function; this function is piece-wise constant and can be written as a linear combination of step functions with the coefficients provided by the shadows.

The challenge now is to define in an appropriate sense how one can approximate all the aspects of the theory by means of this constant by pieces functions. Then the strategy is that, for any given scale, one can define an effective theory by approximating the kinetic operator by a combination of the translation operators that shift between the vertices of the given decomposition, in other words by a periodic function in $p$. As a result one has a set of effective theories at given scales which are mutually related by coarse graining maps. This framework was developed in [6]. For the convenience of the reader we briefly recall part of that framework.

Let us denote the kinematic polymer Hilbert space at the scale $C_{n}$ as $\mathcal{H}_{C_{n}}$, and its basis elements as $e_{\alpha_{i}, C_{n}}$, where $\alpha_{i}=\left[i a_{n},(i+1) a_{n}\right) \in C_{n}$. By construction this basis is orthonormal. The basis elements in the dual Hilbert space $\mathcal{H}_{C_{n}}^{*}$ are denoted by $\omega_{\alpha_{i}, C_{n}}$; they are also orthonormal. The states $\omega_{\alpha_{i}, C_{n}}$ have a simple action on Cyl, $\omega_{\alpha_{i}, C_{n}}\left(\delta_{x_{0}, q}\right)=\chi_{\alpha_{i}, C_{n}}\left(x_{0}\right)$. That is, if $x_{0}$ is in the interval $\alpha_{i}$ of $C_{n}$ the result is one and it is zero if it is not there.

Given any $m \leq n$, we define $d_{m, n}^{*}: \mathcal{H}_{C_{n}}^{*} \rightarrow \mathcal{H}_{C_{m}}^{*}$ as the 'coarse graining' map between the dual Hilbert spaces, that sends the part of the elements of the dual basis to zero while keeping the information of the rest: $d_{m, n}^{*}\left(\omega_{\alpha_{i}, C_{n}}\right)=\omega_{\beta_{j}, C_{m}}$ if $i=j 2^{n-m}$, in the opposite case $d_{m, n}^{*}\left(\omega_{\alpha_{i}, C_{n}}\right)=0$.

At every scale the corresponding effective theory is given by the hamiltonian $H_{n}$. These Hamiltonians will be treated as quadratic forms, $h_{n}: \mathcal{H}_{C_{n}} \rightarrow \mathbb{R}$, given by

$$
h_{n}(\psi)=\lambda_{C_{n}}^{2}\left(\psi, H_{n} \psi\right)
$$

where $\lambda_{C_{n}}^{2}$ is a normalizaton factor. We will see later that this rescaling of the inner product is necessary in order to guarantee the convergence of the renormalized theory. The completely renormalized theory at this scale is obtained as

$$
h_{m}^{\mathrm{ren}}:=\lim _{C_{n} \rightarrow \mathbb{R}} d_{m, n}^{\star} h_{n}
$$

and the renormalized Hamiltonians are compatible with each other, in the sense that

$$
d_{m, n}^{\star} h_{n}^{\text {ren }}=h_{m}^{\text {ren }}
$$

In order to analyze the conditions for the convergence in (28) let us express the Hamiltonian in terms of its eigen-covectors end eigenvalues. We will work with effective Hamiltonians that have a purely discrete spectrum (labelled by $\nu$ ) $H_{n} \cdot \Psi_{\nu, C_{n}}=E_{\nu, C_{n}} \Psi_{\nu, C_{n}}$. We shall also introduce, as an intermediate step, a cut-off in the energy levels. The origin of this cut-off is in the approximation of the Hamiltonian of our system at a given scale with a Hamiltonian of a periodic system in a regime of small energies, as we explained earlier. Thus, we can write

$$
h_{m}^{\nu_{\text {cut }- \text { off }}}=\sum_{\nu=0}^{\nu_{\text {cut }- \text { off }}} E_{\nu, C_{m}} \Psi_{\nu, C_{m}} \otimes \Psi_{\nu, C_{m}}
$$

where the eigen covectors $\Psi_{\nu, C_{m}}$ are normalized according to the inner product rescaled by $\frac{1}{\lambda_{C_{n}}^{2}}$, and the cutoff can vary up to a scale dependent bound, $\nu_{\text {cut-off }} \leq$ $\nu_{\max }\left(C_{m}\right)$. The Hilbert space of covectors together with such inner product will be called $\mathcal{H}_{C_{m}}^{\star \text { ren }}$.

In the presence of a cut-off, the convergence of the microscopically corrected Hamiltonians, equation (28) is equivalent to the existence of the following two limits. The first one is the convergence of the energy levels,

$$
\lim _{C_{n} \rightarrow \mathbb{R}} E_{\nu, C_{n}}=E_{\nu}^{\mathrm{ren}}
$$

Second is the existence of the completely renormalized eigen covectors,

$$
\lim _{C_{n} \rightarrow \mathbb{R}} d_{m, n}^{\star} \Psi_{\nu, C_{n}}=\Psi_{\nu, C_{m}}^{\mathrm{ren}} \in \mathcal{H}_{C_{m}}^{\star \mathrm{ren}} \subset \mathrm{Cyl}^{\star}
$$

We clarify that the existence of the above limit means that $\Psi_{\nu, C_{m}}^{\text {ren }}\left(\delta_{x_{0}, q}\right)$ is well defined for any $\delta_{x_{0}, q} \in$ Cyl. Notice that this point-wise convergence, if it can take place 
at all, will require the tuning of the normalization factors $\lambda_{C_{n}}^{2}$.

Now we turn to the question of the continuum limit of the renormalized covectors. First we can ask for the existence of the limit

$$
\lim _{C_{n} \rightarrow \mathbb{R}} \Psi_{\nu, C_{n}}^{\mathrm{ren}}\left(\delta_{x_{0}, q}\right)
$$

for any $\delta_{x_{0}, q} \in \mathrm{Cyl}$. When this limits exists there is a natural action of the eigen covectors in the continuum limit. Below we consider another notion of the continuum limit of the renormalized eigen covectors.

When the completely renormalized eigen covectors exist, they form a collection that is $d^{\star}$-compatible, $d_{m, n}^{\star} \Psi_{\nu, C_{n}}^{\mathrm{ren}}=\Psi_{\nu, C_{m}}^{\mathrm{ren}}$. A sequence of $d^{\star}$-compatible normalizable covectors define an element of $\overleftarrow{\mathcal{H}}_{\mathbb{R}}^{\star \text { ren }}$, which is the projective limit of the renormalized spaces of covectors

$$
\overleftarrow{\mathcal{H}}_{\mathbb{R}}^{\star \mathrm{ren}}:={\overleftarrow{\lim _{n} \rightarrow \mathbb{R}}}_{\mathcal{H}_{C_{n}}^{\star \mathrm{ren}}}
$$

The inner product in this space is defined by

$$
\left(\left\{\Psi_{C_{n}}\right\},\left\{\Phi_{C_{n}}\right\}\right)_{\mathbb{R}}^{\mathrm{ren}}:=\lim _{C_{n} \rightarrow \mathbb{R}}\left(\Psi_{C_{n}}, \Phi_{C_{n}}\right)_{C_{n}}^{\mathrm{ren}} .
$$

The natural inclusion of $\mathcal{C}_{0}^{\infty}$ in $\overleftarrow{\mathcal{H}}_{\mathbb{R}}^{\star \text { ren }}$ is by an antilinear map which assigns to any $\Psi \in \mathcal{C}_{0}^{\infty}$ the $d^{\star}$-compatible collection $\Psi_{C_{n}}^{\text {shad }}:=\sum_{\alpha_{i}} \omega_{\alpha_{i}} \bar{\Psi}\left(L\left(\alpha_{i}\right)\right) \in \mathcal{H}_{C_{n}}^{\star \mathrm{ren}} \subset \mathrm{Cyl}^{\star}$; $\Psi_{C_{n}}^{\text {shad }}$ will be called the shadow of $\Psi$ at scale $C_{n}$ and acts in Cyl as a piecewise constant function. Clearly other types of test functions like Schwartz functions are also naturally included in $\overleftarrow{\mathcal{H}}_{\mathbb{R}}^{\star \text { ren }}$. In this context a shadow is a state of the effective theory that approximates a state in the continuum theory.

Since the inner product in $\overleftarrow{\mathcal{H}}_{\mathbb{R}}^{\star \text { ren }}$ is degenerate, the physical Hilbert space is defined as

$$
\begin{gathered}
\mathcal{H}_{\text {phys }}^{\star}:=\overleftarrow{\mathcal{H}}_{\mathbb{R}}^{\star \text { ren }} / \operatorname{ker}(\cdot, \cdot)_{\mathbb{R}}^{\text {ren }} \\
\mathcal{H}_{\text {phys }}:=\mathcal{H}_{\text {phys }}^{\star \star}
\end{gathered}
$$

The nature of the physical Hilbert space, whether it is isomorphic to the Schrödinger Hilber space, $\mathcal{H}_{s}$, or not, is determined by the normalization factors $\lambda_{C_{n}}^{2}$ which can be obtained from the conditions asking for compatibility of the dynamics of the effective theories at different scales. The dynamics of the system under consideration selects the continuum limit.

Let us now return to the definition of the Hamiltonian in the continuum limit. First consider the continuum limit of the Hamiltonian (with cut-off) in the sense of its point-wise convergence as a quadratic form. It turns out that if the limit of equation (32) exists for all the eigencovectors allowed by the cut-off, we have $h_{\mathbb{R}}^{\nu_{\text {cut }- \text { off }} \text { ren }}: \mathcal{H}_{\text {poly }, x} \rightarrow \mathbb{R}$ defined by

$$
h_{\mathbb{R}}^{\nu_{\text {cut }- \text { off ren }}}\left(\delta_{x_{0}, q}\right):=\lim _{C_{n} \rightarrow \mathbb{R}} h_{n}^{\nu_{\text {cut }- \text { off }} \text { ren }}\left(\left[\delta_{x_{0}, q}\right]_{C_{n}}\right)
$$

This Hamiltonian quadratic form in the continuum can be coarse grained to any scale and, as can be expected, it yields the completely renormalized Hamiltonian quadratic forms at that scale. However, this is not a completely satisfactory continuum limit because we can not remove the auxiliary cut-off $\nu_{\text {cut-off. If we tried, as }}$ we include more and more eigencovectors in the Hamiltonian the calculations done at a given scale would diverge and doing them in the continuum is just as divergent. Below we explore a more successful path.

We can use the renormalized inner product to induce an action of the cut-off Hamiltonians on $\overleftarrow{\mathcal{H}}_{\mathbb{R}}^{\star \text { ren }}$

$$
h_{\mathbb{R}}^{\nu_{\text {cut }- \text { off ren }}}\left(\left\{\Psi_{C_{n}}\right\}\right):=\lim _{C_{n} \rightarrow \mathbb{R}} h_{n}^{\nu_{\text {cut }- \text { off ren }}}\left(\left(\Psi_{C_{n}}, \cdot\right)_{C_{n}}^{\text {ren }}\right),
$$

where we have used the fact that $\left(\Psi_{C_{n}}, \cdot\right)_{C_{n}}^{\text {ren }} \in \mathcal{H}_{C_{n}}$. The existence of this limit is trivial because the renormalized Hamiltonians are finite sums and the limit exists term by term.

These cut-off Hamiltonians descend to the physical Hilbert space

$$
h_{\mathbb{R}}^{\nu_{\text {cut }- \text { off }} \text { ren }}\left(\left[\left\{\Psi_{C_{n}}\right\}\right]\right):=h_{\mathbb{R}}^{\nu_{\text {cut }- \text { off }} \text { ren }}\left(\left\{\Psi_{C_{n}}\right\}\right)
$$

for any representative $\left\{\Psi_{C_{n}}\right\} \in\left[\left\{\Psi_{C_{n}}\right\}\right] \in \mathcal{H}_{\text {phys }}^{\star}$.

Finally we can address the issue of removal of the cutoff. The Hamiltonian $h_{\mathbb{R}}^{\text {ren }}: \overleftarrow{\mathcal{H}}_{\mathbb{R}}^{\star \text { ren }} \rightarrow \mathbb{R}$ is defined by the limit

$$
h_{\mathbb{R}}^{\text {ren }}:=\lim _{\nu_{\text {cut }- \text { off } \rightarrow \infty}} h_{\mathbb{R}}^{\nu_{\text {cut }- \text { off ren }}}
$$

when the limit exists. Its corresponding Hermitian form in $\mathcal{H}_{\text {phys }}$ is defined whenever the above limit exists. This concludes our presentation of the main results of [6]. Let us now consider several examples of systems for which the continuum limit can be investigated.

\section{EXAMPLES}

In this section we shall develop several examples of systems that have been treated with the polymer quantization. These examples are simple quantum mechanical systems, such as the simple harmonic oscillator and the free particle, as well as a quantum cosmological model known as loop quantum cosmology.

\section{A. The Simple Harmonic Oscillator}

In this part, let us consider the example of a Simple Harmonic Oscillator (SHO) with parameters $m$ and $\omega$, classically described by the following Hamiltonian

$$
H=\frac{1}{2 m} p^{2}+\frac{1}{2} m \omega^{2} x^{2} .
$$

Recall that from these parameters one can define a length scale $D=\sqrt{\hbar / m \omega}$. In the standard treatment one uses 
this scale to define a complex structure $J_{D}$ (and an inner product from it), as we have described in detail that uniquely selects the standard Schrödinger representation.

At scale $C_{n}$ we have an effective Hamiltonian for the Simple Harmonic Oscillator (SHO) given by

$$
H_{C_{n}}=\frac{\hbar^{2}}{m a_{n}^{2}}\left[1-\cos \frac{a_{n} p}{\hbar}\right]+\frac{1}{2} m \omega^{2} x^{2} .
$$

If we interchange position and momentum, this Hamiltonian is exactly that of a pendulum of mass $m$, length $l$ and subject to a constant gravitational field $g$ :

$$
\hat{H}_{C_{n}}=-\frac{\hbar^{2}}{2 m l^{2}} \frac{d^{2}}{d \theta^{2}}+m g l(1-\cos \theta)
$$

where those quantities are related to our system by,

$$
l=\frac{\hbar}{m \omega a_{n}}, \quad g=\frac{\hbar \omega}{m a_{n}}, \quad \theta=\frac{p a_{n}}{\hbar}
$$

That is, we are approximating, for each scale $C_{n}$ the SHO by a pendulum. There is, however, an important difference. From our knowledge of the pendulum system, we know that the quantum system will have a spectrum for the energy that has two different asymptotic behaviors, the SHO for low energies and the planar rotor in the higher end, corresponding to oscillating and rotating solutions respectively ${ }^{2}$. As we refine our scale and both the length of the pendulum and the height of the periodic potential increase, we expect to have an increasing number of oscillating states (for a given pendulum system, there is only a finite number of such states). Thus, it is justified to consider the cut-off in the energy eigenvalues, as discussed in the last section, given that we only expect a finite number of states of the pendulum to approximate SHO eigenstates. With these consideration in mind, the relevant question is whether the conditions for the continuum limit to exist are satisfied. This question has been answered in the affirmative in [6]. What was shown there was that the eigen-values and eigen functions of the discrete systems, which represent a discrete and non-degenerate set, approximate those of the continuum, namely, of the standard harmonic oscillator when the inner product is renormalized by a factor $\lambda_{C_{n}}^{2}=1 / 2^{n}$. This convergence implies that the continuum limit exists as we understand it. Let us now consider the simplest possible system, a free particle, that has nevertheless the particular feature that the spectrum of the energy is continuous.

\footnotetext{
2 Note that both types of solutions are, in the phase space, closed. This is the reason behind the purely discrete spectrum. The distinction we are making is between those solutions inside the separatrix, that we call oscillating, and those that are above it that we call rotating.
}

\section{B. Free Polymer Particle}

In the limit $\omega \rightarrow 0$, the Hamiltonian of the Simple Harmonic oscillator (35) goes to the Hamiltonian of a free particle and the corresponding time independent Schrödinger equation, in the $p$-polarization, is given by

$$
\left[\frac{\hbar^{2}}{m a_{n}^{2}}\left(1-\cos \frac{a_{n} p}{\hbar}\right)-E_{C_{n}}\right] \tilde{\psi}(p)=0
$$

where we now have that $p \in S^{1}$, with $p \in\left(-\frac{\pi \hbar}{a_{n}}, \frac{\pi \hbar}{a_{n}}\right)$. Thus, we have

$$
E_{C_{n}}=\frac{\hbar^{2}}{m a_{n}^{2}}\left(1-\cos \frac{a_{n} p}{\hbar}\right) \leq E_{C_{n}, \max } \equiv 2 \frac{\hbar^{2}}{m a_{n}^{2}} .
$$

At each scale the energy of the particle we can describe is bounded from above and the bound depends on the scale. Note that in this case the spectrum is continuous, which implies that the ordinary eigenfunctions of the Hilbert are not normalizable. This imposes an upper bound in the value that the energy of the particle can have, in addition to the bound in the momentum due to its "compactification".

Let us first look for eigen-solutions to the time independent Schrödinger equation, that is, for energy eigenstates. In the case of the ordinary free particle, these correspond to constant momentum plane waves of the form $e^{ \pm\left(\frac{i p x}{\hbar}\right)}$ and such that the ordinary dispersion relation $p^{2} / 2 m=E$ is satisfied. These plane waves are not square integrable and do not belong to the ordinary Hilbert space of the Schrödinger theory but they are still useful for extracting information about the system. For the polymer free particle we have,

$$
\tilde{\psi}_{C_{n}}(p)=c_{1} \delta\left(p-P_{C_{n}}\right)+c_{2} \delta\left(p+P_{C_{n}}\right)
$$

where $P_{C_{n}}$ is a solution of the previous equation considering a fixed value of $E_{C_{n}}$. That is,

$$
P_{C_{n}}=P\left(E_{C_{n}}\right)=\frac{\hbar}{a_{n}} \arccos \left(1-\frac{m a_{n}^{2}}{\hbar^{2}} E_{C_{n}}\right)
$$

The inverse Fourier transform yields, in the ' $x$ representation',

$$
\begin{aligned}
\psi_{C_{n}}\left(x_{j}\right) & =\frac{1}{\sqrt{2 \pi}} \int_{-\pi \hbar / a_{n}}^{\pi \hbar / a_{n}} \tilde{\psi}(p) e^{\frac{i a_{n}}{\hbar} p j} \mathrm{~d} p= \\
& =\frac{\hbar \sqrt{2 \pi}}{a_{n}}\left(c_{1} e^{i x_{j} P_{C_{n}} / \hbar}+c_{2} e^{-i x_{j} P_{C_{n}} / \hbar}\right)
\end{aligned}
$$

with $x_{j}=a_{n} j$ for $j \in \mathbb{Z}$. Note that the eigenfunctions are still delta functions (in the $p$ representation) and thus not (square) normalizable with respect to the polymer inner product, that in the $p$ polarization is just given by the ordinary Haar measure on $S^{1}$, and there is no quantization of the momentum (its spectrum is still truly continuous). 
Let us now consider the time dependent Schrödinger equation,

$$
i \hbar \partial_{t} \tilde{\Psi}(p, t)=\hat{H} \cdot \tilde{\Psi}(p, t) .
$$

Which now takes the form,

$$
\frac{\partial}{\partial t} \tilde{\Psi}(p, t)=\frac{-i \hbar}{m a_{n}}\left(1-\cos \left(a_{n} p / \hbar\right)\right) \tilde{\Psi}(p, t)
$$

that has as its solution,

$$
\tilde{\Psi}(p, t)=e^{-\frac{i \hbar}{m a_{n}}\left(1-\cos \left(a_{n} p / \hbar\right)\right) t} \tilde{\psi}(p)=e^{\left(-i E_{C_{n}} / \hbar\right) t} \tilde{\psi}(p)
$$

for any initial function $\tilde{\psi}(p)$, where $E_{C_{n}}$ satisfy the dispersion relation (36). The wave function $\Psi\left(x_{j}, t\right)$, the $x_{j}$-representation of the wave function, can be obtained for any given time $t$ by Fourier transforming with (37) the wave function $\tilde{\Psi}(p, t)$.

In order to check out the convergence of the microscopically corrected Hamiltonians we should analyze the convergence of the energy levels and of the proper covectors. In the limit $n \rightarrow \infty, E_{C_{n}} \rightarrow E=p^{2} / 2 m$ so we can be certain that the eigen-values for the energy converge (when fixing the value of $p$ ). Let us write the proper covector as $\Psi_{C_{n}}=\left(\psi_{C_{n}}, \cdot\right)_{C_{n}}^{\text {ren }} \in \mathcal{H}_{C_{n}}^{\star r e n}$. Then we can bring microscopic corrections to scale $C_{m}$ and look for convergence of such corrections

$$
\Psi_{C_{m}}^{\mathrm{ren}} \doteq \lim _{n \rightarrow \infty} d_{m, n}^{\star} \Psi_{C_{n}} .
$$

It is easy to see that given any basis vector $e_{\alpha_{i}} \in \mathcal{H}_{C_{m}}$ the following limit

$$
\Psi_{C_{m}}^{\mathrm{ren}}\left(e_{\alpha_{i}, C_{m}}\right)=\lim _{C_{n} \rightarrow \infty} \Psi_{C_{n}}\left(d_{n, m}\left(e_{\alpha_{i}, C_{m}}\right)\right)
$$

exists and is equal to

$$
\Psi_{C_{m}}^{\text {shad }}\left(e_{\alpha_{i}, C_{m}}\right)=\left[d^{\star} \Psi^{\mathrm{Schr}}\right]\left(e_{\alpha_{i}, C_{m}}\right)=\Psi^{\mathrm{Schr}}\left(i a_{m}\right)
$$

where $\Psi_{C_{m}}^{\text {shad }}$ is calculated using the free particle Hamiltonian in the Schrödinger representation. This expression defines the completely renormalized proper covector at the scale $C_{m}$.

\section{Polymer Quantum Cosmology}

In this section we shall present a version of quantum cosmology that we call polymer quantum cosmology. The idea behind this name is that the main input in the quantization of the corresponding mini-superspace model is the use of a polymer representation as here understood. Another important input is the choice of fundamental variables to be used and the definition of the Hamiltonian constraint. Different research groups have made different choices. We shall take here a simple model that has received much attention recently, namely an isotropic, homogeneous FRW cosmology with $k=0$ and coupled to a massless scalar field $\varphi$. As we shall see, a proper treatment of the continuum limit of this system requires new tools under development that are beyond the scope of this work. We will thus restrict ourselves to the introduction of the system and the problems that need to be solved.

The system to be quantized corresponds to the phase space of cosmological spacetimes that are homogeneous and isotropic and for which the homogeneous spatial slices have a flat intrinsic geometry ( $k=0$ condition). The only matter content is a mass-less scalar field $\varphi$. In this case the spacetime geometry is given by metrics of the form:

$$
\mathrm{d} s^{2}=-\mathrm{d} t^{2}+a^{2}(t)\left(\mathrm{d} x^{2}+\mathrm{d} y^{2}+\mathrm{d} z^{2}\right)
$$

where the function $a(t)$ carries all the information and degrees of freedom of the gravity part. In terms of the coordinates $\left(a, p_{a}, \varphi, p_{\varphi}\right)$ for the phase space $\Gamma$ of the theory, all the dynamics is captured in the Hamiltonian constraint

$$
\mathcal{C}:=-\frac{3}{8} \frac{p_{a}^{2}}{|a|}+8 \pi G \frac{p_{\varphi}^{2}}{2|a|^{3}} \approx 0
$$

The first step is to define the constraint on the kinematical Hilbert space to find physical states and then a physical inner product to construct the physical Hilbert space. First note that one can rewrite the equation as:

$$
\frac{3}{8} p_{a}^{2} a^{2}=8 \pi G \frac{p_{\varphi}^{2}}{2}
$$

If, as is normally done, one chooses $\varphi$ to act as an internal time, the right hand side would be promoted, in the quantum theory, to a second derivative. The left hand side is, furthermore, symmetric in $a$ and $p_{a}$. At this point we have the freedom in choosing the variable that will be quantized and the variable that will not be well defined in the polymer representation. The standard choice is that $p_{a}$ is not well defined and thus, $a$ and any geometrical quantity derived from it, is quantized. Furthermore, we have the choice of polarization on the wave function. In this respect the standard choice is to select the $a$-polarization, in which $a$ acts as multiplication and the approximation of $p_{a}$, namely $\sin \left(\lambda p_{a}\right) / \lambda$ acts as a difference operator on wave functions of $a$. For details of this particular choice see [5]. Here we shall adopt the opposite polarization, that is, we shall have wave functions $\Psi\left(p_{a}, \varphi\right)$.

Just as we did in the previous cases, in order to gain intuition about the behavior of the polymer quantized theory, it is convenient to look at the equivalent problem in the classical theory, namely the classical system we would get be approximating the non-well defined observable ( $p_{a}$ in our present case) by a well defined object (made of trigonometric functions). Let us for simplicity choose to replace $p_{a} \mapsto \sin \left(\lambda p_{a}\right) / \lambda$. With this choice we get an effective classical Hamiltonian constraint that 
depends on $\lambda$ :

$$
\mathcal{C}_{\lambda}:=-\frac{3}{8} \frac{\sin \left(\lambda p_{a}\right)^{2}}{\lambda^{2}|a|}+8 \pi G \frac{p_{\varphi}^{2}}{2|a|^{3}} \approx 0
$$

We can now compute effective equations of motion by means of the equations: $\dot{F}:=\left\{F, \mathcal{C}_{\lambda}\right\}$, for any observable $F \in C^{\infty}(\Gamma)$, and where we are using the effective (first order) action:

$$
S_{\lambda}=\int \mathrm{d} \tau\left(p_{a} \dot{a}+p_{\varphi} \dot{\varphi}-N \mathcal{C}_{\lambda}\right)
$$

with the choice $N=1$. The first thing to notice is that the quantity $p_{\varphi}$ is a constant of the motion, given that the variable $\varphi$ is cyclic. The second observation is that $\dot{\varphi}=8 \pi G \frac{p_{\varphi}}{|a|^{3}}$ has the same sign as $p_{\varphi}$ and never vanishes. Thus $\varphi$ can be used as a (n internal) time variable. The next observation is that the equation for $\left(\frac{\dot{a}}{a}\right)^{2}$, namely the effective Friedman equation, will have a zero for a non-zero value of $a$ given by

$$
a^{*}=\frac{32 \pi G}{3} \lambda^{2} p_{\varphi}^{2}
$$

This is the value at which there will be bounce if the trajectory started with a large value of $a$ and was contracting. Note that the 'size' of the universe when the bounce occurs depends on both the constant $p_{\varphi}$ (that dictates the matter density) and the value of the lattice size $\lambda$. Here it is important to stress that for any value of $p_{\varphi}$ (that uniquely fixes the trajectory in the $\left(a, p_{a}\right)$ plane), there will be a bounce. In the original description in terms of Einstein's equations (without the approximation that depends on $\lambda$ ), there in no such bounce. If $\dot{a}<0$ initially, it will remain negative and the universe collapses, reaching the singularity in a finite proper time. What happens within the effective description if we refine the lattice and go from $\lambda$ to $\lambda_{n}:=\lambda / 2^{n}$ ? The only thing that changes, for the same classical orbit labelled by $p_{\varphi}$, is that the bounce occurs at a 'later time' and for a smaller value of $a^{*}$ but the qualitative picture remains the same.

This is the main difference with the systems considered before. In those cases, one could have classical trajectories that remained, for a given choice of parameter $\lambda$, within the region where $\sin (\lambda p) / \lambda$ is a good approximation to $p$. Of course there were also classical trajectories that were outside this region but we could then refine the lattice and find a new value $\lambda^{\prime}$ for which the new classical trajectory is well approximated. In the case of the polymer cosmology, this is never the case: Every classical trajectory will pass from a region where the approximation is good to a region where it is not; this is precisely where the 'quantum corrections' kick in and the universes bounces.

Given that in the classical description, the 'original' and the 'corrected' descriptions are so different we expect that, upon quantization, the corresponding quantum theories, namely the polymeric and the Wheeler-DeWitt will be related in a non-trivial way (if at all).
In this case, with the choice of polarization and for a particular factor ordering we have,

$$
\left[\left(\frac{1}{\lambda} \sin \left(\lambda p_{a}\right) \frac{\partial}{\partial p_{a}}\right)^{2}+\frac{32 \pi}{3} \ell_{p}^{2} \frac{\partial^{2}}{\partial \varphi^{2}}\right] \cdot \Psi\left(p_{a}, \varphi\right)=0
$$

as the Polymer Wheeler-DeWitt equation.

In order to approach the problem of the continuum limit of this quantum theory, we have to realize that the task is now somewhat different than before. This is so given that the system is now a constrained system with a constraint operator rather than a regular non-singular system with an ordinary Hamiltonian evolution. Fortunately for the system under consideration, the fact that the variable $\varphi$ can be regarded as an internal time allows us to interpret the quantum constraint as a generalized Klein-Gordon equation of the form

$$
\frac{\partial^{2}}{\partial \varphi^{2}} \Psi=\Theta_{\lambda} \cdot \Psi
$$

where the operator $\Theta_{\lambda}$ is 'time independent'. This allows us to split the space of solutions into 'positive and negative frequency', introduce a physical inner product on the positive frequency solutions of this equation and a set of physical observables in terms of which to describe the system. That is, one reduces in practice the system to one very similar to the Schrödinger case by taking the positive square root of the previous equation: $\frac{\partial}{\partial \varphi} \Psi=\sqrt{\Theta}_{\lambda} \cdot \Psi$. The question we are interested is whether the continuum limit of these theories (labelled by $\lambda$ ) exists and whether it corresponds to the WheelerDeWitt theory. A complete treatment of this problem lies, unfortunately, outside the scope of this work and will be reported elsewhere 12 .

\section{DISCUSSION}

Let us summarize our results. In the first part of the article we showed that the polymer representation of the canonical commutation relations can be obtained as the limiting case of the ordinary Fock-Schrödinger representation in terms of the algebraic state that defines the representation. These limiting cases can also be interpreted in terms of the naturally defined coherent states associated to each representation labelled by the parameter $d$, when they become infinitely 'squeezed'. The two possible limits of squeezing lead to two different polymer descriptions that can nevertheless be identified, as we have also shown, with the two possible polarizations for an abstract polymer representation. This resulting theory has, however, very different behavior as the standard one: The Hilbert space is non-separable, the representation is unitarily inequivalent to the Schrödinger one, and natural operators such as $\hat{p}$ are no longer well defined. This particular limiting construction of the polymer theory can shed some light for more complicated systems such as field theories and gravity. 
In the regular treatments of dynamics within the polymer representation, one needs to introduce some extra structure, such as a lattice on configuration space, to construct a Hamiltonian and implement the dynamics for the system via a regularization procedure. How does this resulting theory compare to the original continuum theory one had from the beginning? Can one hope to remove the regulator in the polymer description? As they stand there is no direct relation or mapping from the polymer to a continuum theory (in case there is one defined). As we have shown, one can indeed construct in a systematic fashion such relation by means of some appropriate notions related to the definition of a scale, closely related to the lattice one had to introduce in the regularization. With this important shift in perspective, and an appropriate renormalization of the polymer inner product at each scale one can, subject to some consistency conditions, define a procedure to remove the regulator, and arrive to a Hamiltonian and a Hilbert space.

As we have seen, for some simple examples such as a free particle and the harmonic oscillator one indeed recovers the Schrödinger description back. For other systems, such as quantum cosmological models, the answer is not as clear, since the structure of the space of classical solutions is such that the 'effective description' intro- duced by the polymer regularization at different scales is qualitatively different from the original dynamics. A proper treatment of these class of systems is underway and will be reported elsewhere [12].

Perhaps the most important lesson that we have learned here is that there indeed exists a rich interplay between the polymer description and the ordinary Schrödinger representation. The full structure of such relation still needs to be unravelled. We can only hope that a full understanding of these issues will shed some light in the ultimate goal of treating the quantum dynamics of background independent field systems such as general relativity.

\section{Acknowledgments}

We thank A. Ashtekar, G. Hossain, T. Pawlowski and P. Singh for discussions. This work was in part supported by CONACyT U47857-F and 40035-F grants, by NSF PHY04-56913, by the Eberly Research Funds of Penn State, by the AMC-FUMEC exchange program and by funds of the CIC-Universidad Michoacana de San Nicolás de Hidalgo.
[1] R. Beaume, J. Manuceau, A. Pellet and M. Sirugue, "Translation Invariant States In Quantum Mechanics," Commun. Math. Phys. 38, 29 (1974); W. E. Thirring and H. Narnhofer, "Covariant QED without indefinite metric," Rev. Math. Phys. 4, 197 (1992); F. Acerbi, G. Morchio and F. Strocchi, "Infrared singular fields and nonregular representations of canonical commutation relation algebras", J. Math. Phys. 34, 899 (1993); F. Cavallaro, G. Morchio and F. Strocchi, "A generalization of the Stone-von Neumann theorem to non-regular representations of the CCR-algebra", Lett. Math. Phys. 47 307 (1999); H. Halvorson, "Complementarity of Representations in quantum mechanics", Studies in History and Philosophy of Modern Physics 3545 (2004).

[2] A. Ashtekar, S. Fairhurst and J.L. Willis, "Quantum gravity, shadow states, and quantum mechanics", Class. Quant. Grav. 201031 (2003) arXiv:gr-qc/0207106.

[3] K. Fredenhagen and F. Reszewski, "Polymer state approximations of Schrödinger wave functions", Class. Quant. Grav. 236577 (2006) arXiv:gr-qc/0606090.

[4] M. Bojowald, "Loop quantum cosmology", Living Rev. Rel. 8, 11 (2005) arXiv:gr-qc/0601085]; A. Ashtekar, M. Bojowald and J. Lewandowski, "Mathematical structure of loop quantum cosmology", Adv. Theor. Math. Phys. 7233 (2003) arXiv:gr-qc/0304074; A. Ashtekar, T. Pawlowski and P. Singh, "Quantum nature of the big bang: Improved dynamics" Phys. Rev. D $\mathbf{7 4} 084003$ (2006) arXiv:gr-qc/0607039

[5] V. Husain and O. Winkler, "Semiclassical states for quantum cosmology" Phys. Rev. D 75024014 (2007)
arXiv:gr-qc/0607097; V. Husain V and O. Winkler, "On singularity resolution in quantum gravity", Phys. Rev. D 69084016 (2004). arXiv:gr-qc/0312094].

[6] A. Corichi, T. Vukasinac and J.A. Zapata. "Hamiltonian and physical Hilbert space in polymer quantum mechanics", Class. Quant. Grav. 241495 (2007) arXiv:gr-qc/0610072

[7] A. Corichi and J. Cortez, "Canonical quantization from an algebraic perspective" (preprint)

[8] A. Corichi, J. Cortez and H. Quevedo, "Schrödinger and Fock Representations for a Field Theory on Curved Spacetime", Annals Phys. (NY) 313446 (2004) arXiv:hep-th/0202070.

[9] E. Manrique, R. Oeckl, A. Weber and J.A. Zapata, "Loop quantization as a continuum limit" Class. Quant. Grav. 233393 (2006) arXiv:hep-th/0511222; E. Manrique, R. Oeckl, A. Weber and J.A. Zapata, "Effective theories and continuum limit for canonical loop quantization" (preprint)

[10] D.W. Chiou, "Galileo symmetries in polymer particle representation", Class. Quant. Grav. 24, 2603 (2007) arXiv:gr-qc/0612155.

[11] W. Rudin, Fourier analysis on groups, (Interscience, New York, 1962)

[12] A. Ashtekar, A. Corichi, P. Singh, "Contrasting LQC and WDW using an exactly soluble model" (preprint); A. Corichi, T. Vukasinac, and J.A. Zapata, "Continuum limit for quantum constrained system" (preprint). 